\section{Audio analgesia: Lack of a cross-masking effect on taste*}

\author{
DENNIS McFADDEN†, E. ANNE BARR, and RICHARD E. YOUNG†† \\ University of Texas at Austin, Austin, Texas 78712
}

The effects of intense acoustic stimulation on the sense of taste were examined in two experiments. In the first, a yes-no psychophysical method was used to obtain psychometric functions for the discriminability of weak solutions of $\mathrm{NaCl}$ or sucrose from distilled water. The functions for $\mathrm{NaCl}$ were about 10 times steeper than those for sucrose. In the second experiment, suprathreshold concentrations of these substances were used in conjunction with a magnitude-estimation technique. With neither technique were there any systematic differences in the data taken with and without audio analgesia.

Intense acoustic stimulation has been reported to have analgesic power adequate to allow its being used as the sole analgesic agent in certain dental operations. Gardner and Licklider (1959), Gardner, Licklider, and Weisz (1960), and Gardner (1961) argued that the acoustic stimulation was acting not just as a source of distraction or as an exotic placebo, but that it also was initiating a direct suppressive effect on the pain. Other researchers tend to minimize the possibility of any direct analgesic component, however, and instead attribute the effectiveness of audio analgesia to such "psychological" factors as distraction and/or suggestion.

If Gardner is correct in assuming a direct suppressive component of some kind, then it is possible that this suppression will act not only on pain perception, but also on perception in other modalities as well. That is, if there is cross-modality masking of pain by auditory stimulation, might not there be cross-masking in some other modalities by that same auditory stimulation? The present experiment was designed to examine the effects of intense auditory stimulation on the sense of taste.

It is clear that in the dentist's office, the only situation in which audio analgesia is generally acknowledged to be effective, the effect is on suprathreshold, not threshold, sensations. That is, strong (and aversive) sensations are reduced in magnitude (and, thereby, in aversiveness) by the audio analgesia. Some audio analgesia experiments seem to assume that a decrease in

* This research was supported in part by USPHS Grant NS 08754 from the National Institute of Neurological Diseases and Stroke. Preliminary drafts of the paper were read and criticized by $R . K$. Gummerman and R. P. Erickson. The authors wish to thank Donna Johnson, George Goldstein, and Sam Shimek for their assistance.

tAddress: Department of Psychology. Mezes Hall, University of Texas, Austin, Texas 78712 .

+ Now at the University of California at Santa Barbara. suprathreshold sensation magnitudes will necessarily be accompanied by a simultaneous rise in the perceptual threshold for that sensory dimension, and as a consequence, these studies deal exclusively with threshold-level sensations. This is a curious assumption, however, for an increase in threshold is logieally not a necessary concomitant of a diminution in Thus, any experiment designed to assess the effects of audio analgesia ought to examine its effects on both threshold and suprathreshold sensation magnitudes. This was accomplished in the present study by conducting two experiments. In the first, the Ss were asked to discriminate between distilled water and very weak concentrations of $\mathrm{NaCl}$ or sucrose; in the second, they were asked to make numerical estimates of the magnitude of the sensations associated with various stronger concentrations of the same two substances.

\section{EXPERIMENT 1:}

\section{PSYCHOMETRIC FUNCTIONS}

\section{Procedure}

The data for this experiment were collected with the forced-choice procedure that has come to be known as the yes-no method. With this method there is no determination of a "threshold" as such, but instead the S's ability to discriminate between the presence and the absence of the target stimulus (the "signal") is measured for each of several stimulus intensities. By selecting intensities that lead to performance between chance and perfection, one obtains the data necessary to plot a psychometric function. Forced-choice methods are preferable to classical threshold methods because they allow the $\mathrm{E}$ to obtain relatively independent measures of the S's response criterion and of his ability to discriminate between the two stimulus alternatives (see Egan \& Clarke, 1966, and/or Green \& Swets, psychophysical techniques).

$S$ was seated on one side of a suprathreshold sensation magnitudes. 1966, for discussions of modern wooden partition with a small slot cut in the bottom, and $\mathbf{E}$ sat on the other side. Every $20 \mathrm{sec}, \mathrm{E}$ passed a $3-\mathrm{oz}$ paper cup through the slot to $S$. The cup contained a $10-\mathrm{ml}$ sample of either distilled water or distilled water plus a small quantity of solute $(\mathrm{NaCl}$ for some Ss, sucrose for others). S sipped the sample, held it in his mouth for approximately $5 \mathrm{sec}$, and then spit it into a large bottle at his side. $S$ then responded, "Yes, that was a signal trial," or, "No, that was distilled water," by passing one of two small cards through the slot toward E. E recorded the response, and then passed through the slot a similar card which indicated whether the trial had been a signal or a no-signal trial; that is, $\mathbf{S}$ received trial-by-trial feedback about the correctness of his responses. Response and feedback took approximately $10 \mathrm{sec}$; at the end of this time, $\mathbf{S}$ rinsed his mouth for about $5 \mathrm{sec}$ with approximately $20 \mathrm{ml}$ of distilled water, spit it into the large bottle, and then immediately took the next cup from $E$. A clock with a sweep second hand was used by $S$ as an aid in keeping to this schedule of $20 \mathrm{sec}$ per trial.

A block of trials consisted of 20 trials, and a daily session consisted of four blocks. Between blocks there were rest periods lasting $\mathbf{2 - 5} \mathrm{min}$. For all four blocks of a session, all signal trials were of the same molarity, and the molarities were varied from session to session in a pseudorandom order. Approximately 5-10 min before a block of trials was run, a second $E$ prepared a tray of 20 cups according to the master protocol; in this way, both $S$ and $E$ were kept ignorant of the molarity being tested in any given session. The sequence of trials within each block was made up from a table of random numbers, the only constraint being that there could not be more than five consecutive trials of one type. The a priori probability of a signal trial was 0.5 , and the Ss were informed of this fact. Prior to the first block of the day, $S$ was given five signal trials at the molarity to be used during that session; prior to each of the last three blocks of a session, only one such familiarization trial was given. The Ss were instructed not to eat, drink, or smoke for at least $1 \mathrm{~h}$ prior to the daily session, and an attempt was made to run each $S$ at approximately the same time every day.

The water used in this experiment, both for mixing samples and for rinsing, was kept at room temperature. Temperature readings of individual samples were taken throughout the experiment; these readings ranged from $20^{\circ}$ to $24^{\circ} \mathrm{C}$ across blocks, but the range was always less than $1^{\circ} \mathrm{C}$ within a block. 


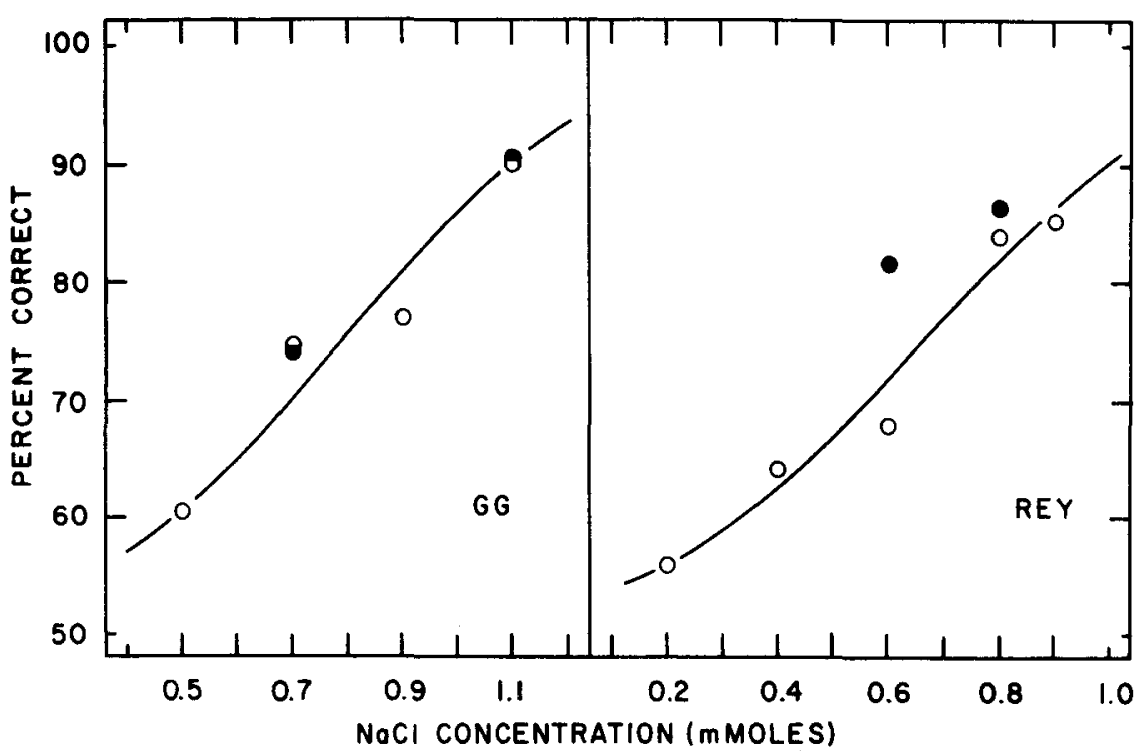

Fig. 1. Psychometric functions for $\mathrm{NaCl}$ obtained with the yes-no method. Each point is based upon 240 trials. The ogives shown were derived from the normal curve. Open symbols are the data taken without audio analgesia, and solid symbols are those taken with analgesia.

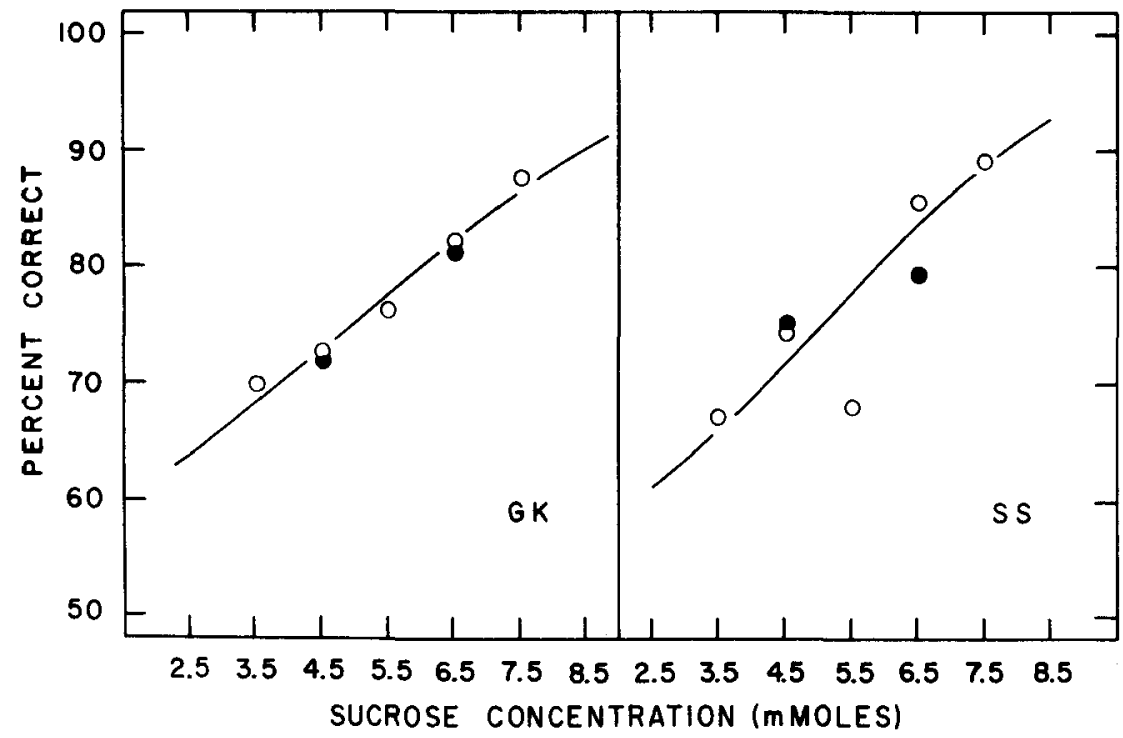

Fig. 2. Psychometric functions for sucrose obtained with the yes-no method. Each point is based upon 240 trials. The ogives shown were derived from the normal curve. Open symbols are the data taken without audio analgesia, and solid symbols are those taken with analgesia.

TDH-39 headphones mounted in moulded-rubber ear cushions (Grason-Stadler 001) were worn only on those blocks of trials for which audio analgesia was scheduled. $\mathbf{S}$ turned on the noise himself by closing a switch immediately prior to rinsing his mouth, and he turned it off after he spit out the $10-\mathrm{ml}$ sample. Thus, the noise was on for about $10 \mathrm{sec}$ of the 20-sec trial, and it was on for about 5 sec prior to the S's tasting the sample. This procedure of intermittent acoustic stimulation was adopted so as to minimize the possibility of acoustic trauma. The noise was obtained from a of trials, the version of proportion correct called $\mathrm{P}(\mathrm{C})_{2}$ (see McFadden, 1970) was computed, and a psychometric function was plotted for each $S$ by averaging the 12 proportions correct obtained for each molarity.

The Ss in both Experiments 1 and 2 were undergraduate psychology majors who were paid on an hourly basis.

Results and Discussion

The psychometric functions for four of the Ss who participated in this experiment are shown in Figs. 1 and 2. The ordinate is the percentage of correct detections, and the abscissa is concentration in millimoles ( $\mathrm{mM}$ ) plotted linearly. The curves are normal ogives that were fitted to the data by eye.

If $75 \%$ or $80 \%$ correct detections is taken as a kind of "threshold," then the sensitivity of these Ss for both $\mathrm{NaCl}$ and sucrose is equal to or better than that of any reported by Pfaffmann (1959) or McBurney and Pfaffmann (1963). Clearly, the data taken with analgesia (solid symbols) differ little from those taken without analgesia (open symbols); the only exception, REY, actually performed better with analgesia than without.

Data were taken for three other Ss, but they are not shown because it was felt that they would contribute little. One $S$ had a particularly ragged psychometric function for $\mathrm{NaCl}$, and two others were run only without analgesia. One of the latter deserves comment. In order to perform better than the chance level, this $\mathrm{S}$ required $\mathrm{NaCl}$ concentrations of $1.5,2.5,3.5$, and $4.5 \mathrm{mM}$. That is, her psychometric function was much lower in slope and was displaced toward much higher concentrations than the functions of any of the other Ss. Except for this, her data appear to be perfectly regular, implying that the discrepancy reflects a real difference in sensitivity. When this $\mathbf{S}$ was questioned, she admitted to using large quantities of salt on her food.

Of the many variables that are important in taste experiments, among the most important is the state of adaptation of the taste receptors. McBurney and Pfaffmann (1963) showed that threshold sensitivity for $\mathrm{NaCl}$ was critically dependent on the salinity of the solution with which their Ss' tongues were bathed, and, further, that normal saliva was saline enough to elevate threshold concentration by between one and two orders of magnitude from its value when the tongue is bathed by distilled water. In the present experiment, it was not feasible to bathe the tongue continuously with distilled water between samples, so the $S s$ were impressed with the importance of 
careful rinsing. The Ss were instructed to "swish" the $20-\mathrm{ml}$ rinse around for at least 5 sec before spitting it out and then to taste the sample immediately. This rinsing procedure must have been effective, because all but one of our Ss would have been $100 \%$ correct at $2.2 \mathrm{mM}$, the $\mathrm{NaCl}$ threshold McBurney and Pfaffmann obtained with their rinsing procedure (p. 528). Presumably the rinse was equally effective in minimizing the effects of saliva on sucrose sensitivity.

While the data in Figs. 1 and 2 may not be quite as regular as those typically obtained with the yes-no technique, as for example in auditory masking experiments, they are, nevertheless, good enough to establish the feasibility of the technique for use with taste stimuli. To our knowledge, this experiment is the first extension of the yes-no technique to the taste modality. Pangborn, Chrisp, and Bertolero (1970) used a similar task, paired comparisons, and plotted percent correct against $\mathrm{NaCl}$ concentration, but the data were averaged across Ss, and very few trials were taken on each $S$. Even so, those data are in reasonable agreement with the present data.

One of the advantages of using a forced-choice technique rather than one that yields a single, "threshold" value can be illustrated by comparing Figs. 1 and 2. These figures show two things. The first, the fact that human sensitivity for $\mathrm{NaCl}$ is about seven or eight times greater than that for sucrose, could be about equally well established with a threshold technique. The second, however, the spectacular difference in the rates at which detection performance improves with small increases in the concentrations of the two substances, would remain unknown if the data were collected with a threshold technique. Note that the abscissa in Fig. 1 is expanded by a factor of about 10 relative to that in Fig. 2, meaning that the $\mathrm{NaCl}$ functions would be nearly vertical lines if they were plotted on the same scale as the sucrose functions. To our knowledge, this enormous difference in the slopes of the psychometric functions for $\mathrm{NaCl}$ and sucrose is reported here for the first time.

\section{EXPERIMENT 2}

\section{MAGNITUDE ESTIMATION}

\section{Procedure}

In this experiment the magnitude-estimation technique developed by Stevens was used to assess the effects of audio analgesia on suprathreshold taste sensations. With this technique, $\mathrm{S}$ is given a set of relatively strong stimuli and is asked to assign a number proportional to the magnitude of the sensation aroused by

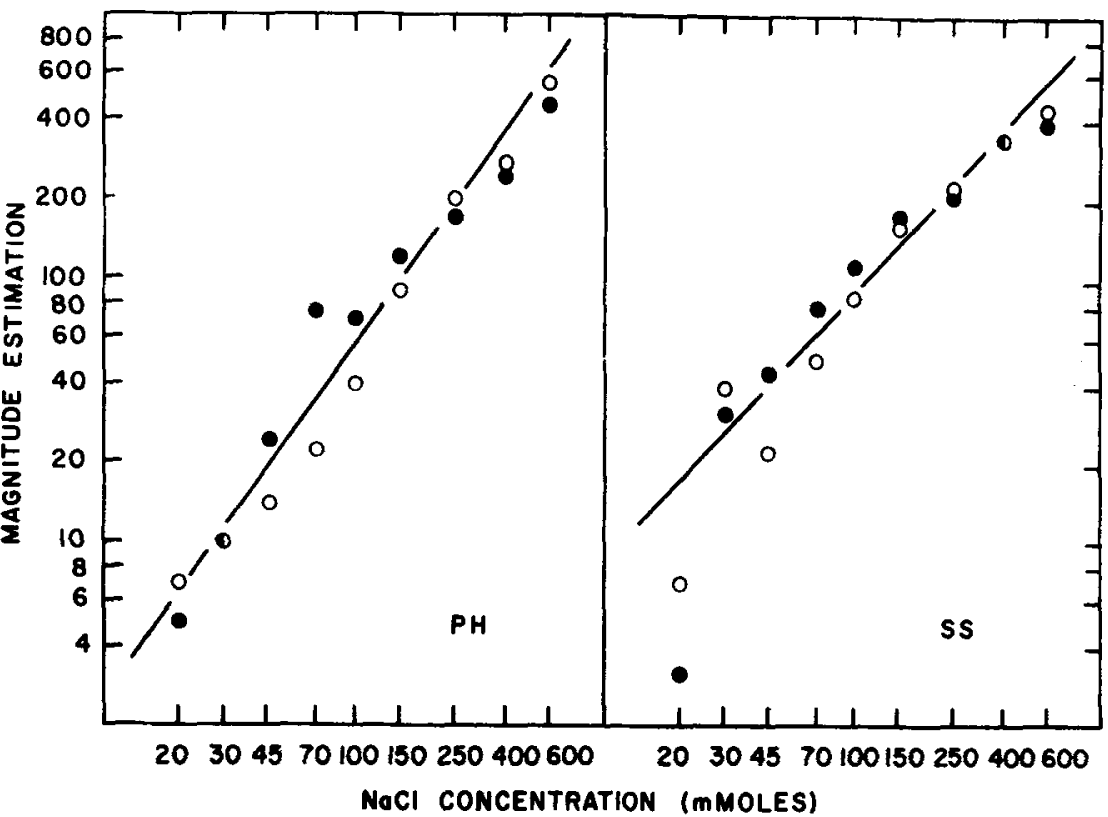

Fig. 3. Magnitude-estimation data for NaCl. The points are geometric means of two judgments. Both ordinate and abscissa are plotted logarithmically. The slope of the line fitted to the data of PH is 1.34 ; for SS it is 1.02 . Open symbols are the data taken without audio analgesia, and solid symbols are those taken with analgesia.

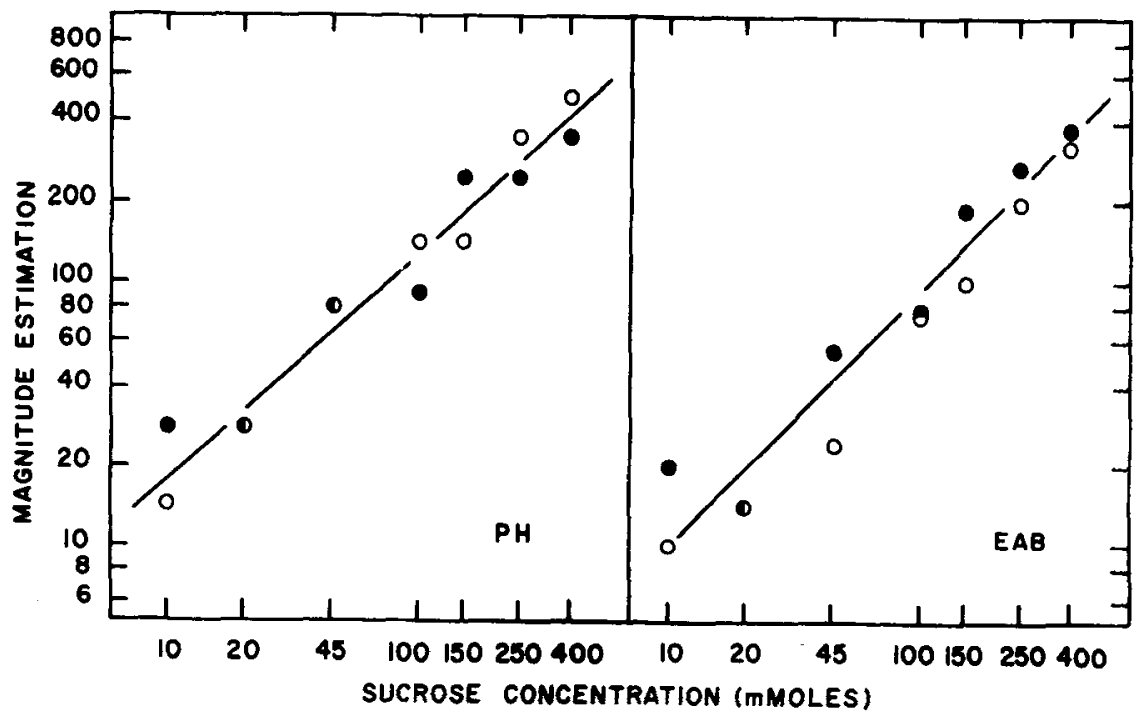

Fig. 4. Magnitude-estimation data for sucrose. The points are geometric means of two judgments. Both ordinate and abscissa are plotted logarithmically. The slope of the line fitted to the data of $P H$ is 0.86 ; for $E A B$ it is 0.96 . Open symbols are the data taken without audio analgesia, and solid symbols are those taken with analgesia.

each. Typical outcomes of such experiments are data that are linear when plotted in a log-log coordinate system (Stevens, 1961).

The physical arrangement of $S$ and $E$ was identical to that in Experiment 1. Again, each trial took approximately $20 \mathrm{sec}-5 \mathrm{sec}$ of rinse, followed immediately by $5 \mathrm{sec}$ of tasting, followed by $10 \mathrm{sec}$ for responding and getting ready for the next trial. Unlike Experiment 1, responses were made orally and there was no trial-by-trial feedback. When analgesia was employed, $S$ turned the noise on about 5 sec prior to tasting the sample, and he turned it off after spitting out the sample. Each block consisted of two trials for each molarity. The molarities were presented in a scrambled order, the only constraint being that no molarity could be presented on two consecutive trials. Prior to each block, S tasted a $100-\mathrm{mM}$ solution and was told to call the sensory magnitude associated with 
it "100." If the block was scheduled to be run with analgesia, $S$ tasted the 100-mM standard with the noise on. All noise parameters were the same as in Experiment 1.

Because the magnitude-estimation experiment required so little time reiative to the detection experiment, six Ss were run on both $\mathrm{NaCl}$ and sucrose, both with and without analgesia. One data session was required to obtain all the data for $\mathrm{NaCl}$, and one for sucrose. On the day preceding each data session, there was a training session during which $\mathrm{S}$ was generally familiarized with the stimuli and the task-he read the instructions to Ss published by Stevens (1969) and he received three blocks of trials, two without analgesia and one with. In the data sessions, there were also three blocks of trials. The first block was run without analgesia; these data were regarded as practice and were discarded. The second and third blocks were the data blocks, one run with analgesia and one without. For both $\mathrm{NaCl}$ and sucrose, the set of molarities used was the same for all Ss and for both the practice and the data sessions; nine molarities were used for $\mathrm{NaCl}$ and seven for sucrose.

\section{Results and Discussion}

In general, the data for individual Ss are quite ragged, and when fitted with straight lines, the data points tend to fall below the line either at the high or at the low concentrations, and sometimes at both. The data for three of the best $\mathrm{Ss}$ in this experiment are shown in Figs. 3 and 4 . The data points are geometric means of the two magnitude estimates made for each molarity. The open symbols are the data taken without analgesia and the solid symbols are those taken with analgesia. The straight lines were simply fitted to the data by eye. Had there been an analgesia effect, the data taken with analgesia would have had a lower slope than the data taken without analgesia; however, for no $S$ were there any systematic differences in the two sets of data.

Stevens (1969) has reported magnitude-estimation data for both $\mathrm{NaCl}$ and sucrose. Our highest concentrations were much weaker than those of Stevens, partly because our Ss found his highest concentrations aversive. The slope of the straight line fitted by Stevens to his $\mathrm{NaCl}$ data varied between 1.3 and 1.5 ; in the present experiment the slopes for individual Ss varied between 0.93 and 1.34. For sucrose, Stevens obtained a slope of 1.3 ; the values ranged from 0.55 to 1.24 in the present experiment. That is, for Stevens's Ss, sensory magnitude tended to grow more rapidly as a function of stimulus magnitude than it did for our Ss. The basis for this difference is not clear, but it may be that our smaller range of concentrations produced a regression effect (Stevens \& Greenbaum, 1966) greater than that present in Stevens's experiment. Another difference between the two experiments is that Stevens used tap, not distilled, water.

\section{DISCUSSION}

Most attempts to take audio analgesia out of the dentist's office and bring it into the laboratory have been unsuccessful. Camp, Martin, and Chapman (1962) applied radiant energy to various locations on the skin and found no changes either in pain threshold or in suprathreshold pain discrimination due to the presence of an intense white noise. Carlin, Ward, Gershon, and Ingraham (1962) stimulated teeth electrically and measured the threshold for "tingle" both with and without simultaneous auditory stimulation. They found no differences and concluded that distraction and suggestion were critical factors in the success of audio analgesia in the dentist's office, even though these authors were unable to demonstrate the effectiveness of "suggestion" with their Ss. In contrast, Howitt and Stricker (1966) also used an electrical dental stimulator, and they concluded that children did show higher "tolerance thresholds" if given both auditory stimulation and suggestion, but that "response thresholds" were unchanged by these agents. They, too, emphasized the importance of suggestion in the clinically successful application of audio analgesia.

It is unclear exactly what role "suggestion" played in the present experiments. No attempt was made to manipulate this important variable, but it is certainly the case that all of the Ss knew something about both the phenomenon of audio analgesia and the purpose of the experiments. And, while expectations about the outcome of the experiments were mixed, the outcomes themselves clearly were not.

One obvious criticism of the present experiments is that the noise level used was inadequate to establish analgesic effects, and that this alone accounts for the failure of both experiments. In anticipation of this criticism, it can be said that much pilot work was done with volunteer $S$ s in an attempt to find an effect at higher noise levels (up to about $125 \mathrm{~dB}$ SPL), with both intermittent and continuous noise. These volunteers also experimented, in vain, with several different noise bandwidths because Gardner and Licklider had indicated that the "higher" frequency components were the more effective in reducing pain Regrettably, most audio-analgesia studies are vague about the actual noise levels employed; typically, they report only an overall level with no statement about the noise bandwidth, thereby making it impossible to determine the noise spectrum level. It is likely that the spectrum level used in the present experiments was very similar to that in many other analgesia experiments, even though the overall level was not.

The form of the psychometric function is an important source of information about the nature of the sensory processing underlying performance. Consequently, the great differences observed here in the steepness of the functions for $\mathrm{NaCl}$ and sucrose make the determination of the forms of the functions for other sapid substances an attractive undertaking. It would be nice to be able to compare these differences in slope with data taken from other modalities, but, regrettably, psychometric functions for threshold-level stimuli have not been systematically collected for the various modalities. It can be noted, however, that Egan, Lindner, and McFadden (1969) reported remarkable similarity in the forms of the psychometric functions determined with the same Ss for several different auditory masking conditions.

\section{REFERENCES}

CAMP, W., MARTIN, R., \& CHAPMAN, L. F. Pain threshold and discrimination of pain intensity during brief exposure to intense noise. Science, 1962, 135 , 788-789.

CARLIN, S., WARD, W. D., GERSHON, A., \& INGRAHAM, R. Sound stimulation and its effect on dental sensation threshold, Science, 1962, 138, 1258-1259.

EGAN, J. P., \& CLARKE, F. R. Psychophysics and signal detection. In J. B. Sidowski (Ed.), Experimental methods and instrumentation in psychology. New York: McGraw-Hill, 1966.

EGAN, J. P., LINDNER, W. A., \& MCF ADDEN, D. Masking-level differences and the form of the psychometric function. Perception \& Psychophysics, $1969,6,209-215$.

GARDNER, W. J. Audio analgesia: Theory and practice. International Dental Journal, 1961, 11, 467-474.

GARDNER, W. J., \& LICKLIDER, J. C. R. Auditory analgesia in dental operations. The Journal of the American Dental Association, 1959, 59, 1144-1149.

GARDNER, w. J., LICKLIDER, J. C. R., \& WEISZ, A. Z. Suppression of pain by sound. Science, 1960, 132, 32-33.

GREEN, D. M., \&WETS, J. A. Signal detection theory and psychophysics. New York: Wiley, 1966.

HOWITT, J. W., \& STRICKER, G. Objective evaluation of audio analgesia effects. The Journal of the American Dental Association, 1966, 73, 874.877.

MCBURNEY, D. H., \& PFAFFMANN, C. Gustatory adaptation to saliva and sodium chloride. Journal of Experimental Psychology, 1963, 65, 523-529. 
McFADDEN, D. Three computational versions of proportion correct for use in forced-choice experiments. Perception \& Psychophysics, 1970, 8, 336-342.

PANGBORN, R. M., CHRISP, R. B., \& BERTOLERO, L. L. Gustatory, salivary, and oral thermal responses to solutions of sodium chloride at four temperatures. Perception \& Psychophysics, 1970, 8, 69-75.
PFAFFMANN, $C$. The sense of taste $\ln \mathrm{J}$ Field (Ed.), Handbook of physiology. Vol. 1, Chap. 20. Baltimore: Williams \& Wilkins, 1959

STEVENS, $S$. $S$. The psychophysics of sensory function. In $\mathrm{W}$. A. Rosenblith (Ed.), Sensory communication. Cambridge, Mass: M.I.T. Press, 1961.

STEVENS, S. S. Sensory scales of taste intensity. Perception \& Psychophysics,
$1969,6,302-308$.

STEVENS, S. S., \& GREENBAUM, H. B Regression effect in psychophysical judgment. Perception \& Psychophysics, $1966,1,439-446$.

(Accepted for publication February 15 1971.) 